\title{
Exploring the Potential of RET Kinase Inhibition for Irritable Bowel Syndrome: A Preclinical Investigation in Rodent Models of Colonic Hypersensitivity
}

\author{
John P. Russell, Ehsan Mohammadi, (1) Casey O. Ligon, Anthony C. Johnson, \\ Michael D. Gershon, Meenakshi Rao, Yuhong Shen, Chi-Chung Chan, \\ Hilary S. Eidam, Michael P. DeMartino, Mui Cheung, Allen I. Oliff, Sanjay Kumar, \\ and Beverley Greenwood-Van Meerveld
}

Virtual Proof of Concept Discovery Performance Unit, GlaxoSmithKline, King of Prussia, Pennsylvania (J.P.R., H.S.E., M.P.D., M.C., A.I.O., S.K.); Oklahoma Center for Neuroscience, University of Oklahoma Health Sciences Center, Oklahoma City, Oklahoma (E.M., C.O.L., A.C.J., B.G.-V.M.); Department of Pathology and Cell Biology, College of Physicians and Surgeons (M.D.G.) and Department of Pediatrics (M.R.), Columbia University, New York, New York; and WuXi AppTec Co., Ltd., Waigaoqiao Free Trade Zone, Shanghai, China (Y.S., C.-C.C.)

Received August 16, 2018; accepted November 7, 2018

\begin{abstract}
Abdominal pain represents a significant complaint in patients with irritable bowel syndrome (IBS). While the etiology of IBS is incompletely understood, prior exposure to gastrointestinal inflammation or psychologic stress is frequently associated with the development of symptoms. Inflammation or stress-induced expression of growth factors or cytokines may contribute to the pathophysiology of IBS. Here, we aimed to investigate the therapeutic potential of inhibiting the receptor of glial cell linederived neurotrophic factor, rearranged during transfection (RET), in experimental models of inflammation and stress-induced visceral hypersensitivity resembling IBS sequelae. In RET-cyan fluorescent protein $\left[(\mathrm{CFP}) \mathrm{Ret}^{\mathrm{CFP} /+}\right]$ mice, thoracic and lumbosacral dorsal root ganglia were shown to express RET, which colocalized with calcitonin gene-related peptide. To understand
\end{abstract}

the role of RET in visceral nociception, we employed GSK3179106 as a potent, selective, and gut-restricted RET kinase inhibitor. Colonic hyperalgesia, quantified as exaggerated visceromotor response to graded pressures $(0-60 \mathrm{~mm} \mathrm{Hg})$ of isobaric colorectal distension (CRD), was produced in multiple rat models induced 1) by colonic irritation, 2) following acute colonic inflammation, 3) by adulthood stress, and 4) by early life stress. In all the rat models, RET inhibition with GSK3179106 attenuated the number of abdominal contractions induced by CRD. Our findings identify a role for RET in visceral nociception. Inhibition of RET kinase with a potent, selective, and gut-restricted small molecule may represent a novel therapeutic strategy for the treatment of IBS through the attenuation of post-inflammatory and stress-induced visceral hypersensitivity.

\section{Introduction}

Irritable bowel syndrome (IBS) is a common gastrointestinal (GI) illness with a worldwide prevalence of approximately $5 \%-20 \%$ that is characterized by a constellation of clinical symptoms including abdominal pain and discomfort, bloating, and abnormal bowel habits (Longstreth et al., 2006; Camilleri, 2012; Halland and Talley, 2013). Because the etiology of the disease has not been established, diagnosis is difficult and

The authors affiliated with GlaxoSmithKline have equity interest in GlaxoSmithKline.

This study was supported by a grant from GlaxoSmithKline. This work was supported by the National Institutes of Health National Institute of Neurological Disorders and Stroke (grant R01 NS015547 to M.G.) and the National Institutes of Health National Institute of Diabetes and Digestive and Kidney Diseases (grant K08 DK110532 to M.R.).

https://doi.org/10.1124/jpet.118.252973. relies primarily on the presence of a specific symptom complex (Mayer, 2008). In patients with IBS, the sensory inputs/outputs in the peripheral and central nervous system are altered, resulting in a heightened and disproportionate sensory experience for bowel disturbances termed visceral hypersensitivity (Azpiroz et al., 2007). While it is unclear whether enhancement of central and/or peripheral mechanisms underlies visceral hypersensitivity, there is experimental evidence to support supraspinal, spinal, and enteric mechanisms. For example, sensitization of visceral afferent nociceptors by stress produces visceral hypersensitivity (Camilleri, 2012), while peripheral insults such as an enteric infection can lead to sensitization of the neurons within the enteric nervous system (Spiller and Garsed, 2009). Although the sensitizing event may be transient, its impact on the central nervous system and the enteric nervous system may be long lasting (Brierley and Linden,

ABBREVIATIONS: CFP, cyan fluorescent protein; CGRP, calcitonin gene-related peptide; CRD, colorectal distension; DMSO, dimethylsulfoxide; DRG, dorsal root ganglia; GDNF, glial cell line-derived neurotrophic factor; GI, gastrointestinal; IBS, irritable bowel syndrome; PN, postnatal; RET, rearranged during transfection; RM-ANOVA, repeated-measures analysis of variance; TNBS, 2,4,6-trinitrobenzenesulfonic acid; VMR, visceromotor response; WAS, water avoidance stress. 
2014). In fact, patients with IBS have been shown to have increased nerve fiber density and sprouting in intestinal mucosal tissues; moreover, supernatants from these intestinal tissues can stimulate nerve outgrowth and increase neuronal signaling in vitro, suggesting that neurotrophic factors and/or inflammatory cytokines might be mediators of neuronal plasticity in IBS (Buhner et al., 2012; Brierley and Linden, 2014; Dothel et al., 2015).

The glial cell line-derived neurotrophic factor (GDNF) family ligands, which include GDNF, neurturin, artemin, and persephin bind specifically to one of four coreceptors known as GDNF family receptors alpha 1-4 and the tyrosine kinase receptor, rearranged during transfection (RET) (Airaksinen and Saarma, 2002; Borrello et al., 2013; Ibáñez, 2013; Plaza-Menacho et al., 2014). Together the GDNF family ligands and RET/GDNF family receptor alpha regulate the developing nervous system (Baloh et al., 2000; Sariola and Saarma, 2003). The study of patients with Hirschsprung's disease, who suffer from colonic intestinal pseudo-obstruction due to variable lengths of bowel aganglionosis, often in association with loss-of-function mutations in RET (Edery et al., 1994), has provided insight into the role of RET kinase in the development of the enteric nervous system. Experimental evidence in a conditional knockout of the RET kinase coreceptor, GDNF family receptor alpha 3, showed reduction in visceral hypersensitivity, suggesting that RET kinase may also be important in the signaling of visceral nociception (Tanaka et al., 2011). These observations led us to hypothesize that antagonizing RET signaling in the colon may offer a novel therapeutic approach to treat abdominal pain associated with IBS. Here, we employed a novel, selective, and potent GI-restricted RET kinase inhibitor, GSK3179106 (Schenck Eidam et al., 2018). Having demonstrated that colonic afferent projections from thoracic and lumbosacral dorsal root ganglia (DRG) coexpress RET and calcitonin generelated peptide (CGRP), we built upon these observations by the functional characterization of GSK3179106 on visceral perception using multiple rodent models of colonic hypersensitivity. Taken together, our data suggest that RET kinase activity in the adult gut functions as part of an extrinsic neural pathway regulating colonic sensation and a RET antagonist has potential utility for the treatment of IBS.

\section{Materials and Methods}

Animals. Experiments were performed on male Sprague-Dawley, Fischer 344, or Long-Evans rats (250-350 g) purchased from Charles River Laboratories (Wilmington, MA). Timed-pregnant dams used during early life stress experiments were also purchased from Charles River Laboratories and arrived on gestational day E09 and were single housed on aspen shavings. Upon arrival or after weaning, male rats were housed two per cage with free access to food and water at $21-23^{\circ} \mathrm{C}$ and a 12 -hour light/dark cycle within the University of Oklahoma Health Sciences Center Department of Comparative Medicine's animal facility in Oklahoma City, OK. Ret ${ }^{\mathrm{CFP} /+}$ mice (Uesaka et al., 2008) were handled and housed in accordance with the Institutional Animal Care and Use Committee guidelines for Columbia University Medical Center. All animals were acclimated to facility housing for a minimum of 1 week before experimentation. To further minimize experimental stress, the rats were brought to the laboratory for an additional week to acclimate to the laboratory environment and animal handlers. All experimental procedures were approved by the University of Oklahoma Health Sciences Center Institutional Animal Care and Use Committee (Protocol numbers:
14-083-H and 14-171-H) or the Columbia University Institutional Animal Care and Use Committee (Protocol number: AC-AAAM5550) and in accordance with the GlaxoSmithKline Policy on the Care, Welfare and Treatment of Laboratory Animals. In the current studies, female rats were excluded due to estrus cycle effects on GI function, which remain incompletely understood.

Detection of RET-Expressing DRG Neurons. Ret ${ }^{\mathrm{CFP} /+}$ mice used for immunohistochemistry have been described previously (Uesaka et al., 2008). Immunohistochemical staining of mouse DRG was performed using Rabbit Anti-CGRP at 1:1000 (Immunostar, Hudson, WI) and Chicken Anti-GFP at 1:1000 (Aves Laboratories, Tigard, OR) primary antibodies according to a published protocol (Rao et al., 2015).

In Vitro Inhibition of Human and Rat RET Enzyme. The effect of GSK3179106 on human and rat RET enzymatic activity and motesanib on human RET enzymatic activity was determined in an in vitro biochemical homogeneous time-resolved fluorescence assay (Schenck Eidam et al., 2018).

Jump Dilution Assay. The effect of GSK3179106 on human RET enzymatic activity was determined in an in vitro biochemical homogeneous time-resolved fluorescence jump dilution assay to determine the dissociation rate of the compound from the RET kinase. Incubation solutions were prepared as follows: solution A [10 nM RET enzyme in $1 \%$ dimethylsulfoxide (DMSO)] and solution B (10 nM RET enzyme, $5.3 \mathrm{nM}$ GSK3179106 $\left(10 \mathrm{X} \mathrm{IC}_{50}\right)$ in $\left.1 \% \mathrm{DMSO}\right)$. The solutions were incubated at $23^{\circ} \mathrm{C}$ for 1 hour. For each time point the following four reactions were run: 1 ) no inhibitor control: $8 \mu \mathrm{l}$ solution $\mathrm{A}+784 \mu \mathrm{l}$ substrate solution (10 $\mu \mathrm{M}$ ATP, $10 \mathrm{mM} \mathrm{MgCl}_{2}, 0.5 \mathrm{mM}$ dithiothreitol, $0.5 \mu \mathrm{M}$ Cisbio TK biotin-peptide substrate in $1 \mathrm{X}$ assay buffer $)+8 \mu \mathrm{l}$ DMSO; 2) RET enzyme $+0.1 \mathrm{X}$ inhibitor control: $8 \mu$ l solution $\mathrm{A}+$ $784 \mu \mathrm{l}$ substrate solution $+8 \mu \mathrm{l} 5.3 \mathrm{nM}$ GSK3179106 $\left.\left(10 \mathrm{X} \mathrm{IC} \mathrm{IC}_{50}\right) ; 3\right)$ RET enzyme $+0.1 \mathrm{X}$ inhibitor dilution: $8 \mu \mathrm{l}$ solution $\mathrm{B}+784 \mu \mathrm{l}$ substrate solution $+8 \mu \mathrm{l}$ DMSO; and 4) RET enzyme $+10 \mathrm{X}$ inhibitor control: $8 \mu \mathrm{l}$ solution $\mathrm{B}+784 \mu \mathrm{l}$ substrate solution $+8 \mu \mathrm{l} 530 \mathrm{nM}$ GSK3179106 (1000X $\left.\mathrm{IC}_{50}\right)$. A time course was created such that the four reactions were stopped every 10 minutes for the first hour, every 15 minutes for the second hour, and then every 20 minutes from hours $2-6$ by transferring $10 \mu \mathrm{l}$ of each reaction to a well containing $5 \mu \mathrm{l}$ of $75 \mathrm{mM}$ EDTA solution. Then, $5 \mu \mathrm{l} /$ well of homogeneous time-resolved fluorescence detection mix buffer (25 mM EDTA, 100X dilution of Ab europium cryptate and $125 \mathrm{nM}$ streptavidin-XL665 in $50 \mathrm{mM}$ HEPES $\mathrm{pH}$ 7.0) was added and the plate was incubated for 1 hour at $23^{\circ} \mathrm{C}$. The fluorescence emission was measured at $590 \mathrm{~nm}$ (cryptate) and $665 \mathrm{~nm}$ (XL665) for human RET and the ratio (665/590) was calculated for each well.

Cell-Based RET Phosphorylation Enzyme-Linked Immunosorbent Assay. The effect of GSK3179106 and motesanib on RET phosphorylation in a human medullary thyroid carcinoma cell line, TT, or a human neuroblastoma cell line, SK-N-AS, was determined in an in vitro enzyme-linked immunosorbent assay with all antibodies used at a dilution of 1:1000 (Schenck Eidam et al., 2018). The SK-N-AS cells were maintained in $10 \%$ fetal bovine serum, $1 \mathrm{X}$ nonessential amino acid, $1 \%$ sodium pyruvate, and $1 \mathrm{X}$ penicillin/streptomycin in Dulbecco's modified Eagle's medium. In the case of SK-N-AS cells only, the cells were stimulated with $80 \mathrm{ng} / \mathrm{ml}$ GDNF for 2 hours to activate RET kinase.

Proliferation Assay. The effect of GSK3179106 on TT, SK-N-AS, and A549 cell growth was determined in an in vitro proliferation assay. The effect of motesanib on TT and A549 cell growth was determined in an in vitro proliferation assay. The TT, SK-N-AS, or A549 cells were plated into 96 -well plates at $6000,10,000$, or 2000 cells per well in $50 \mu \mathrm{l}$ culture media and incubated overnight at $37^{\circ} \mathrm{C}$ in a $5 \% \mathrm{CO}_{2}$ incubator. On day 0, GSK3179106 was diluted from $10 \mathrm{mM}$ in DMSO using $4 \mathrm{X}$ dilution over nine points for TT and SK-N-AS cells and from $3 \mathrm{mM}$ using $3 \mathrm{X}$ dilution over nine points for A549 cells. A compound plate was prepared by the addition of $5 \mu \mathrm{l}$ of diluted GSK3179106 or DMSO to a 96-well polypropylene V bottom plate to which $250 \mu \mathrm{l} /$ well of culture media was added. The plated cells were 
then treated with $50 \mu \mathrm{l}$ of diluted compound such that the final starting concentration of GSK3179106 was $10 \mu \mathrm{M}$ and cells were incubated either 8 days for TT cells or 3 days for SK-N-AS and A549 cells at $37^{\circ} \mathrm{C}$ in the $5 \% \mathrm{CO}_{2}$ incubator. After incubation, cells were lysed with $50 \mu \mathrm{l} /$ well of CellTiter-Glo reagent (Promega, Madison, WI) and luminescence was measured on Envision (PerkinElmer, Waltham, MA).

Assessment of Colonic Sensitivity and Colonic Compliance. Colonic sensitivity was assessed by measuring the visceromotor response (VMR) to colorectal distension (CRD) by visual counting of the number of abdominal contractions in response to increasing levels of CRD (0-60 $\mathrm{mm} \mathrm{Hg}$ ). On the day of colonic sensitivity assessment, a balloon $(5 \mathrm{~cm})$ catheter was inserted $10 \mathrm{~cm}$ into the colon. The catheter was taped to the base of the tail to hold it in place. The catheter was then connected to a barostat (Distender Series IIR Barostat; G \& J Electronics Inc., Toronto, Canada) for controlled, isobaric inflation of the balloon, and CRD, using constant pressure (isobaric) tonic distension, was conducted at 0, 20, 40, and $60 \mathrm{~mm} \mathrm{Hg}$. Each CRD pressure was maintained for a period of 10 minutes during which time the number of abdominal muscle contractions were counted visually and a 10-minute recovery period was allowed between distensions. The CRD pressures were ordered in a random fashion and the observer was blinded to the treatment. Colonic compliance was determined from using the pressure-volume data generated by the barostat.

Acute Colonic Irritation-Induced Hypersensitivity. Fifty-two male Sprague-Dawley rats $(225-250 \mathrm{~g}, \sim 7$ to 8 weeks old) were purchased from Charles River Laboratories. Rats were housed two per cage under standard conditions of temperature and humidity with a 12: 12-hour light-dark cycle within the Department of Comparative Medicine's animal facility at the University of Oklahoma's Health Sciences Center. Food and water were available ad libitum. All animals were treated as follows for the induction of acute visceral afferent neuron sensitization: 5 minutes after the last dose of vehicle or drug and 1 hour prior to colonic sensitivity assessment, a volume of $1.5 \mathrm{ml}$ dilute $(0.6 \% \mathrm{v} / \mathrm{v})$ acetic acid was infused into the rat colon via a catheter through the anus to the level of the midcolon. Sixty minutes following infusion visceral afferents were sensitized, resulting in acute colonic hypersensitivity. For sham-treated animals, rats received an intracolonic infusion of $1.5 \mathrm{ml}$ of saline instead of acetic acid. Motesanib, a RET antagonist, was used as a positive control for the model (Polverino et al., 2006). One rat did not complete the experiment due to damage to the colon when inserting the colonic balloon catheter.

Postinflammatory Visceral Hypersensitivity. For the induction of postinflammatory visceral hypersensitivity, 70 male SpragueDawley rats (225-250 g, $\sim 7$ to 8 weeks old) were purchased from Charles River Laboratories, and following an overnight fast (14-16 hours) acute colitis was induced by intracolonic infusion of 2,4,6trinitrobenzenesulfonic acid (TNBS) at a concentration of $50 \mathrm{mg} / \mathrm{kg}$ in $25 \%$ ethanol. Rats were then returned to the animal facility to recover spontaneously from the TNBS-induced colitis. Intracolonic administration of TNBS induced acute colitis that lasted approximately 1 week. On day 15 post-TNBS, rats were subjected to a qualifying colonic sensitivity assessment following an overnight fast (14-16 hours) consisting of three $40 \mathrm{~mm} \mathrm{Hg}$ balloon distensions as described previously. Rats that qualified (18 or more abdominal contractions at $40 \mathrm{~mm} \mathrm{Hg}$ balloon pressure for any of the three distensions) were considered hypersensitive and remained in the study $(n=36)$, while rats that did not qualify ( 17 or less abdominal contractions at $40 \mathrm{~mm}$ $\mathrm{Hg}$ for all three distensions) were removed from the study $(n=34)$. Qualifying animals were then subjected to a second colonic sensitivity assessment at post-TNBS day 30 . Historically, approximately $50 \%$ of rats subjected to TNBS-induced colitis develop postinflammatory colonic hypersensitivity that is evident beyond day 30 post-TNBS infusion. The experimenter was not blinded for the qualifying series at day 15 post-TNBS, but was blinded to treatment of the subsequent test at day 30 post-TNBS. Additionally, tegaserod, a $5 \mathrm{HT}_{4}$ agonist, was used as a positive control for the model. Three rats did not complete the experiment due to damage to their colon when inserting the colonic balloon catheter. An additional 14 male Sprague-Dawley rats received a saline enema and were tested at 15 and 30 days postenema to serve as a normosensitive control group.

Chronic Stress-Induced Visceral Hypersensitivity. For the induction of chronic adult stress, 50 male Fischer 344 rats (225-250 g, 10-12 weeks old) were purchased from Charles River Laboratories. Water avoidance stress (WAS) was performed by adding water at $8 \mathrm{~cm}$ of depth into a plastic container $(40 \times 50 \times 32 \mathrm{~cm})$. Rats were then placed onto a plastic platform $(7 \times 7 \times 9 \mathrm{~cm})$ attached to the center of the container surrounded by water. WAS was administered 1 hour per day for 10 consecutive days as a model of chronic psychologic stress $(n=36)$. For sham-treated animals $(n=14)$, rats were placed on the platform in a dry plastic container for 1 hour per day for 10 days. Fecal pellet output of each animal was recorded during each WAS session to ensure animals did not acclimate to the stressor. Twenty-four hours following the final WAS treatment rats were subjected to the measurement of colonic sensitivity. Additionally, fexofenadine, a histamine receptor 1 antagonist, was used as a positive control for the model.

Early Life Stress-Induced Visceral Hypersensitivity. For the induction of early life stress, timed pregnant Sprague-Dawley female rats were purchased from Charles Rivers Laboratories. Female rats arrived approximately on gestational day 9 to allow for acclimation to the animal facility before parturition. The day of birth was assigned as postnatal (PN) day 0 and litters were crossfostered and culled to no more than 12 pups with at least two female pups per litter on PN day 1 . Two rats did not have litters and were euthanized without experimentation.

Limited Nesting Model of Early Life Stress. During limited nesting, dams and the pups were placed in a wire bottom cage and supplied with a single paper towel for nesting material. Male rat pups from each litter were subjected to the limited nesting paradigm on PN days 2-9. Thirty-two male pups were weaned on PN day 21 and housed two per cage by treatment, while female pups were culled on $\mathrm{PN}$ day 21. Limited nesting in male pups results in the development of colonic hypersensitivity in adulthood (PN day 90) (Prusator and Greenwood-Van Meerveld, 2015). An additional series dosed with doxantrazole, a mast cell stabilizer, was used as a positive control. One rat did not complete the experiment due to damage to the colon when inserting the colonic balloon catheter.

Maternal Separation Model of Early Life Stress. Male rat pups from each litter were subjected to the maternal separation paradigm, wherein the pups were removed from the dam and kept on warmed $\left(36-37^{\circ} \mathrm{C}\right)$ bedding in an adjacent room for 180 minutes on $\mathrm{PN}$ days 2-14, weaned on PN day $21(n=12)$, housed in standard rodent cages with two animals per cage, and then subjected to experimentation as an adult between PN days $90-100(n=12)$. Female pups were culled at weaning on PN day 21.

Drug Dosing. For oral dosing, GSK3179106 was prepared as a suspension in $0.5 \%$ hydroxypropyl methylcellulose and $0.1 \%$ Tween 80 at $2.5 \mathrm{ml} / \mathrm{kg}$ and administered twice a day at 8:00 AM and 4:00 PM for 3.5 days for all groups. Dosing solutions were prepared daily and stored in amber bottles at $4^{\circ} \mathrm{C}$. For all treatment groups rats were fed a standard diet ad libitum during the dosing period but were fasted for 14-16 hours prior to the assessment of colonic sensitivity. Motesanib $(10 \mathrm{mg} / \mathrm{kg})$, dosed with the same vehicle and schedule (twice a day, 3.5 days by mouth) was used was a positive control for the acute colonic irritation-induced colonic hypersensitivity model (Polverino et al., 2006). A subset of rats in the chronic stress-induced visceral hypersensitivity group received $6 \mathrm{mg} / \mathrm{kg}$ i.p. doses of the positive control drug fexofenadine, which has been shown to attenuate stress-induced visceral hypersensitivity (Stanisor et al., 2013), dissolved in $10 \%$ ethanol in sterile saline or vehicle at 11:00 AM and 5:00 PM on the final day of WAS and 30 minutes before the colonic sensitivity assessment the next day for a total of three doses equaling $18 \mathrm{mg} / \mathrm{kg}$ over a 24 -hour period. A positive control consisting of the mast cell stabilizer doxantrazole $(10 \mathrm{mg} / \mathrm{kg})$ dissolved in $0.5 \% \mathrm{NaHCO}_{3}$ in sterile saline or vehicle was administered intraperitoneally to a subset of rats exposed to neonatal maternal separation 30 minutes prior to the colonic sensitivity assessment (van den Wijngaard et al., 2009) and a positive control of tegaserod $(1 \mathrm{mg} / \mathrm{kg})$ dissolved in propylene glycol 
TABLE 1

Mean IC $_{50}$ values for GSK3179106 and motesanib against RET kinase in enzyme or cell-based assays

Values are mean $\mathrm{IC}_{50} \pm$ S.D. of at least two experiments.

\begin{tabular}{lcc}
\hline \multicolumn{1}{c}{ Assay } & GSK3179106 & Motesanib \\
\hline & $n M$ & $n M$ \\
Human RET enzyme & $0.4 \pm 0.2^{a}$ & $46.9 \pm 25.4^{a}$ \\
Rat RET enzyme & $0.2 \pm 0.1$ & $\mathrm{NA}$ \\
TT cell phospho-RET & $11.1 \pm 1.1^{a}$ & $559.9 \pm 219.5^{a}$ \\
SK-N-AS cell phospho-RET & $4.6 \pm 1.7$ & $612.2 \pm 197.0$ \\
TT cell proliferation & $25.5 \pm 3.4$ & $1502.0 \pm 540.7$ \\
SK-N-AS cell proliferation & $>10,000$ & $\mathrm{NA}$ \\
A549 cell proliferation & $17,173 \pm 5310^{b}$ & $20,646 \pm 4809^{b}$ \\
\hline
\end{tabular}

NA, not available.

${ }^{a}$ Data previously reported (Schenck Eidam et al., 2018).

${ }^{b}$ Value is mean $\mathrm{IC}_{30} \pm$ S.D. of at least seven experiments.

was administered to a subset of rats in the postinflammatory visceral hypersensitivity cohort 10 minutes prior to colonic hypersensitivity assessment (Greenwood-Van Meerveld et al., 2006).

Drugs and Chemicals. Fexofenadine, tegaserod, doxantrazole, motesanib, and all other reagents were purchased from Sigma-Aldrich (St. Louis, MO), unless otherwise specified. GSK3179106 was provided by GlaxoSmithKline (Schenck Eidam et al., 2018).

Data Analysis. Data from in vitro enzymatic and cell-based assays were analyzed using GraphPad Prism software (GraphPad Software, Inc., La Jolla, CA). Curve fits were applied within GraphPad Prism using a sigmoidal dose-response curve with variable slope. Data for in vivo assays were analyzed using GraphPad Prism software and are presented as mean \pm S.E.M. Two-way repeated-measures analysis of variance (RM-ANOVA) was used to correct for multiple distension pressures in the colonic sensitivity assessment. A Bonferroni's post hoc test was used to compare individual mean values between groups; $P<$ 0.05 was considered statistically significant. All experimenters were blind to treatment of colonic sensitivity assessment. For the colonic sensitivity assessment, $n=$ an individual rat. Animals were randomly assigned to treatment. Group size was based on similar studies.

\section{Results}

Characterization of RET Kinase Inhibitor GSK3179106. To gain an understanding of the role of RET kinase function in regulating visceral nociception, an extensive medicinal chemistry effort led to the discovery of GSK3179106 as a highly potent and selective inhibitor of RET kinase (Schenck Eidam et al., 2018). In an in vitro enzyme assay of rat RET kinase, GSK3179106 exhibited a mean IC $_{50}$ value of $0.2 \mathrm{nM}$, which was similar to the reported mean $\mathrm{IC}_{50}$ value for human of $0.4 \mathrm{nM}$, and demonstrated reversible albeit slow recovery of the human enzymatic activity with a half-life of 234 minutes (Table 1). In cell-based assays, GSK3179106 inhibited RET phosphorylation in SK-N-AS, a neuroblastoma cell line expressing the wild-type RET kinase, and TT cells, a human medullary thyroid carcinoma cell line expressing a constitutively active RET kinase with mean $\mathrm{IC}_{50}$ values of 4.6 and $11.1 \mathrm{nM}$, respectively. Similarly, GSK3179106 inhibited the proliferation of the RETdependent TT cell line with a mean $\mathrm{IC}_{50}$ value of $25.5 \mathrm{nM}$; however, it had no effect on the proliferation of the RETindependent SK-N-AS and A549 cell lines (mean $\mathrm{IC}_{50}>10 \mu \mathrm{M}$ and $\mathrm{IC}_{30}>17 \mu \mathrm{M}$, respectively). In comparison with the known RET inhibitor motesanib, GSK3179106 demonstrated a nearly $100 \times$ increase in potency (Table 1) (Polverino et al., 2006). GSK3179106 demonstrated inhibition of both rat and human RET enzymes and inhibited RET phosphorylation in both RET-expressing cell lines. Furthermore, the growth inhibition observed for the RET-dependent TT cell line and the lack of activity in RET-independent cell-based proliferation assays utilizing SK-N-AS and A549 cell lines provided evidence that GSK3179106 was highly selective for the inhibition of RET kinase and not a general cytotoxic agent.

A Subset of DRG Neurons Coexpressing RET and CGRP Projects to the Colon. To determine whether extrinsic sensory neurons at DRG levels that project to the colon express RET, Ret ${ }^{\mathrm{CFP} /+}$ mice, in which the Ret promoter drives expression of CFP, were used (Uesaka et al., 2008). Many neurons in lower thoracic (Fig. 1, A-C) and lumbosacral (Fig. 1, D-F) DRG demonstrated RET expression in adult mice. Visceral afferent projections from DRG to the colonic mucosa are predominantly immunoreactive for CGRP (Spencer et al., 2014). A subset of RET-expressing DRG neurons in Ret ${ }^{\mathrm{CFP} /+}$ mice colocalized with CGRP immunoreactivity, suggesting that RET may be expressed in visceral afferent neurons.

RET Inhibition with GSK3179106 Normalizes Colonic Hypersensitivity in Rodent Models. Animal models recapitulate many of the symptoms of abdominal pain observed in human IBS and therefore provide a useful tool in the
Ret.DAPI
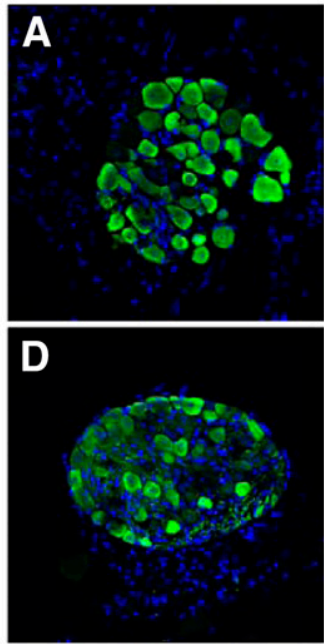

CGRP
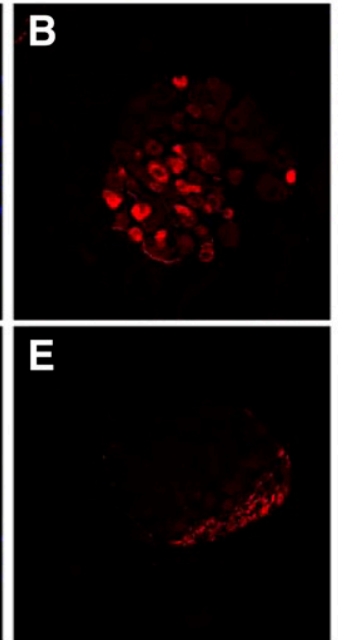

Ret.CGRP.DAPI

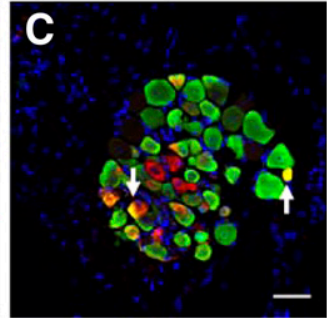

$\mathbf{F}$

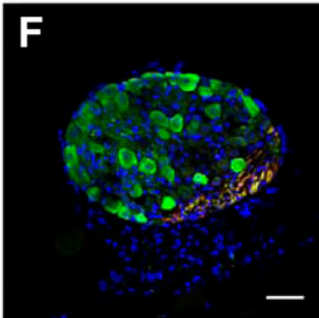

Fig. 1. RET is expressed in peptidergic spinal afferent neurons located in thoracic and lumbosacral DRG of Ret ${ }^{\mathrm{CFP} /+}$ mice. CFP immunoreactivity (green labels) Ret-expressing cells in cross sections of DRG from lower thoracic (T11) (A-C) and lumbosacral (L6) (D-F) levels of an adult Ret ${ }^{\mathrm{CFP} /+}$ mouse. CGRP immunoreactivity (red) (B) colocalizes with CFP in a subset of Ret-expressing neurons (arrows) (C). 4',6Diamidino-2-phenylindole (DAPI) marks nuclei (blue). In this particular cross section of L6 DRG (D-F), note that there are no CGRP-immunoreactive cell bodies (E), but almost all of the CGRP-immunoreactive afferent projections colocalize with CFP (F). Scale bar, $50 \mu \mathrm{m}$. 
understanding of the pathophysiology and effects of therapeutic interventions (Holschneider et al., 2011; Greenwood-Van Meerveld et al., 2015; Moloney et al., 2015).

Acute Colonic Irritation-Induced Hypersensitivity. This rodent model utilizes low concentration acetic acid to induce a transient sensitization of colonic afferents, which upon CRD induces a VMR that can be monitored visually as abdominal contractions, and is used to assess the severity of induced visceral pain (Langlois et al., 1994; Plourde et al., 1997). An increase in the number of abdominal contractions was observed with an acetic acid enema compared with that of a saline enema at colonic distension pressures of 40 or $60 \mathrm{~mm}$ $\mathrm{Hg}$ (Fig. 2A), demonstrating the sensitization of afferent neurons. Specifically, there was a significant effect of enema/ drug treatment $[F(2,19)=10.58 ; P=0.0008]$, a significant effect of colonic distension pressure $[F(3,57)=82.73 ; P<$ $0.0001]$, and a significant interaction between treatment and distension pressure $[F(6,57)=3.13 ; P=0.01](n=7$ to 8 per group; two-factor RM-ANOVA). Treatment with motesanib, a known RET inhibitor, exhibited a decrease in observed
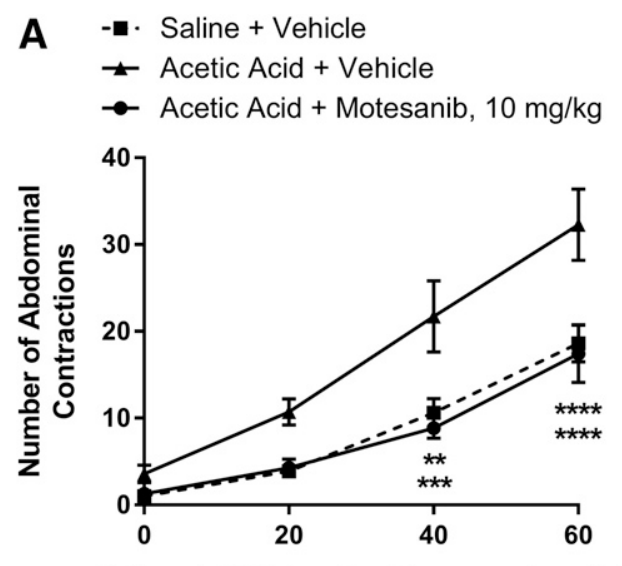

Colorectal Distension Pressure $(\mathrm{mmHg})$ abdominal contractions similar to the use of tegaserod, a known $5 \mathrm{HT}_{4}$ agonist and positive control for the model (Greenwood-Van Meerveld et al., 2006; Polverino et al., 2006). To determine if the effect was specifically due to RET inhibition in the model, a selective RET kinase inhibitor, GSK3179106, when given by oral gavage at 3 and $10 \mathrm{mg} / \mathrm{kg}$ for 3.5 days twice a day, attenuated (33\%-60\% inhibition) the VMR in comparison with sensitized animals dosed with vehicle (Fig. 2B). Similar to the previous results, there was a significant effect of drug treatment $[F(2,18)=27.49 ; P<0.0001]$, a significant effect of colonic distension pressure $[F(3,54)=289.4 ; P<0.0001]$, and a significant interaction between treatment and distension pressure $[F(6,54)=12.67 ; P<0.0001](n=7$ per group; twofactor RM-ANOVA). In contrast to sensitized animals, RET inhibition had little effect on visceral sensitivity in shamtreated animals (Fig. 2C), demonstrated by the lack of a significant effect of drug treatment $[F(1,13)=3.96 ; P=0.07]$ and a nonsignificant interaction between treatment and distension pressure $[F(3,39)=2.40 ; P=0.08]$; however, as expected, there was a significant effect of colonic distension
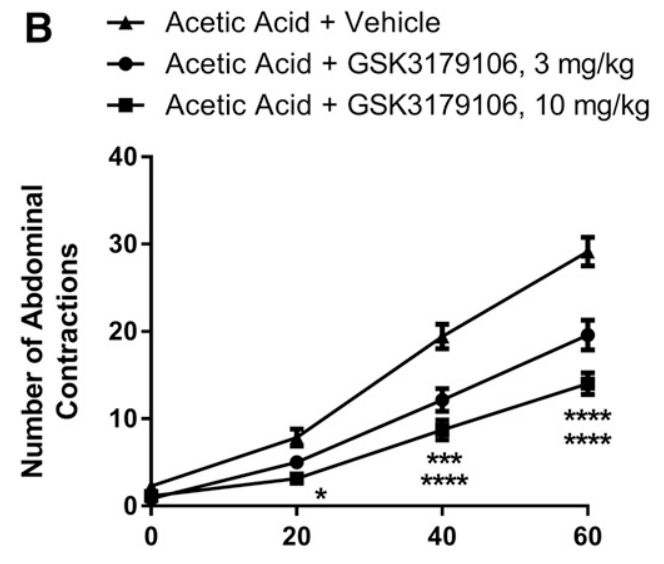

Colorectal Distension Pressure $(\mathrm{mmHg})$
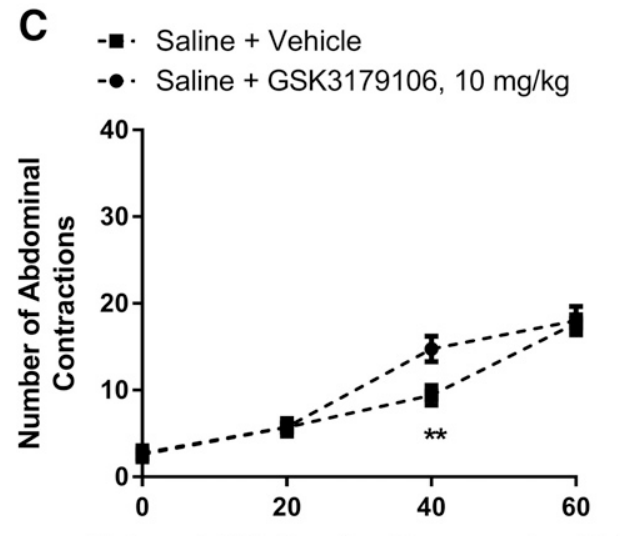

Colorectal Distension Pressure $(\mathrm{mmHg})$

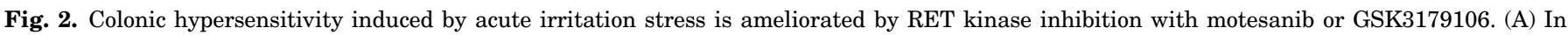

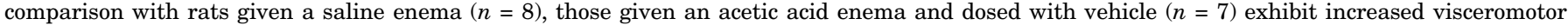

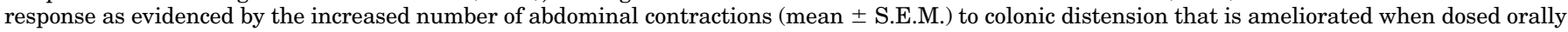

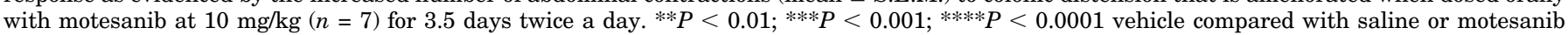

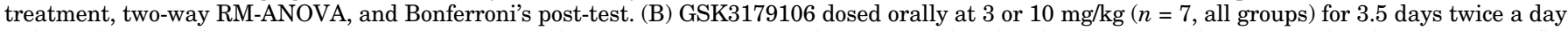

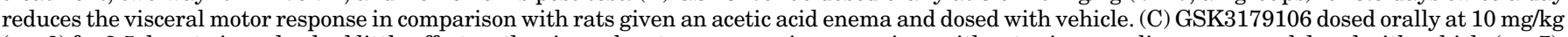

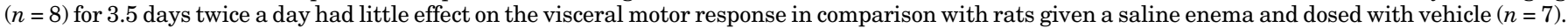
$* P<0.05 ; * * * P<0.01 ; * * * * P<0.0001$ vehicle compared with GSK3179106 treatment, two-way RM-ANOVA, and Bonferroni's post-test. 
pressure $[F(3,39)=66.23 ; P<0.0001](n=7$ or 8 ; two-factor RM-ANOVA). Colon pressure-volume relationships in acetic acid-sensitized colons were similar for animals receiving either vehicle or the RET inhibitor (Fig. 3), with a nonsignificant effect of drug treatment $[F(1,8)=0.08 ; P=0.78]$, a significant effect of colonic distension pressure $[F(3,24)=$ 43.04; $P<0.0001$ ], and a nonsignificant interaction between treatment and distension pressure effect $[F(3,24)=1.53$; $P=0.23]$ ( $n=5$ per group; two-factor RM-ANOVA), demonstrating that RET inhibition had no effect on colonic compliance.

Chronic Postinflammatory and Stress-Induced Visceral Hypersensitivity. Having demonstrated a normalization of noninflammatory irritation-induced colonic hypersensitivity assessed via CRD to induce a VMR and measuring the resulting abdominal contractions, we next examined the effect of RET inhibition on colonic hypersensitivity following a transient colonic inflammation induced by TNBS. GSK3179106 was administered orally at $10 \mathrm{mg} / \mathrm{kg}$ for 3.5 days twice a day and led to 34\%-43\% inhibition in VMR to CRD (Fig. 4A). As anticipated, there was a significant effect of drug treatment $[F(1,15)=23.26$; $P=0.0002$ ], a significant effect of colonic distension pressure $[F(3,45)=266.9 ; P<0.0001]$, and a significant interaction between treatment and distension pressure $[F(3,45)=10.85$; $P<0.0001]$ ( $n=8$ or 9 ; two-factor RM-ANOVA). Similarly, following chronic adult stress induced by water avoidance, a 33\%-35\% inhibition in VMR to CRD was observed upon RET inhibition (Fig. 4B), as shown by a significant effect of drug treatment $[F(1,16)=65.26 ; P<0.0001]$, a significant effect of colonic distension pressure $[F(3,48)=238.6 ; P<0.0001]$, and a significant interaction between treatment and distension pressure $[F(3,48)=10.77 ; P<0.0001](n=9$ per group; twofactor RM-ANOVA). The known pharmacologically efficacious compounds, tegaserod (5- $\mathrm{HT}_{4}$ agonist) and fexofenadine (histamine receptor 1 antagonist), confirmed the performance of the animal models used (Fig. 4, C and D) (Greenwood-Van Meerveld et al., 2006; Stanisor et al., 2013). Specifically, for tegaserod, there was a significant effect of drug treatment $[F(1,14)=61.11 ; P<0.0001]$, a significant effect of colonic distension pressure $[F(3,42)=292.3 ; P<0.0001]$, and a significant interaction between treatment and distension pressure $[F(3,42)=16.42 ; P<0.0001](n=8$ or 9 ; two-factor RM-ANOVA), while for fexofenadine there was a significant effect of drug treatment $[F(1,16)=42.46 ; P<0.0001]$, a

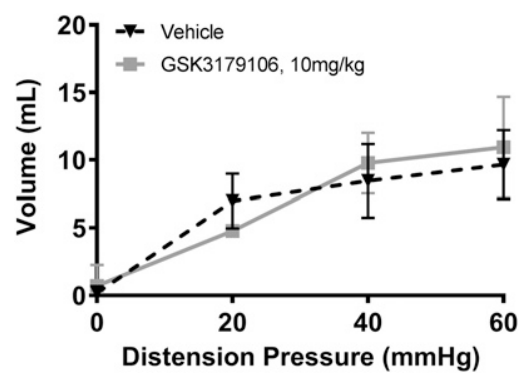

Fig. 3. RET inhibition has no effect on colonic compliance in a model of acute visceral hypersensitivity. In comparison with rats sensitized with acetic acid and dosed orally with vehicle $(n=5)$, rats dosed with $10 \mathrm{mg} / \mathrm{kg}$ GSK3179106 $(n=5)$ demonstrated no difference in the mean colon volumes at $0,20,40$, and $60 \mathrm{~mm} \mathrm{Hg}$ distension pressure determined from barostat measurements. Two-way RM-ANOVA and Bonferonni's post-hoc test for multiple comparisons revealed no statistical difference between vehicle and GSK3179106 treatment. significant effect of colonic distension pressure $[F(3,48)=313.6$; $P<0.0001]$, and a significant interaction between treatment and distension pressure $[F(3,48)=11.59 ; P<0.0001](n=9$ per group; two-factor RM-ANOVA). In contrast to sensitized animals, RET inhibition had no effect on visceral sensitivity in sham-treated animals in both models (Fig. 4, E and F). In the sham postinflammatory model, there was no significant effect of drug treatment $[F(1,12)=1.28 ; P=0.28]$, a significant effect of distension pressure $[F(3,36)=139.0 ; P<0.0001]$, and no significant interaction between treatment and distension pressure $[F(3,36)=1.31 ; P=0.29](n=6$ or 8 ; two-factor RM-ANOVA). In contrast, in the sham WAS model, there was a significant effect of drug treatment $[F(1,12)=13.10 ; P=0.004]$, a significant effect of colonic distension pressure $[F(3,36)=$ 654.6; $P<0.0001$, but no significant interaction between treatment and distension pressure $[F(3,36)=1.81 ; P=0.16]$ ( $n=6$ or 8; two-factor RM-ANOVA).

Early Life Stress-Induced Visceral Hypersensitivity. We next investigated the effect of GSK3179106 on visceral hypersensitivity in adult male rats following exposure to early life stress, modeled in animals through limiting the bedding material to neonates or temporary maternal separation (Schwetz et al., 2005; Gareau et al., 2007; Prusator and Greenwood-Van Meerveld, 2015). In comparison with rats that had developed colonic hypersensitivity after exposure to early life stress induced by limited nesting or maternal separation stress and dosed with vehicle, rats orally dosed with GSK3179106 exhibited a decreased VMR (32\%-42\% inhibition) to colonic distension (Fig. 5, A and B). For the limited nesting model, there was a significant effect of drug treatment $[F(1,13)=30.43 ; P<0.0001]$, a significant effect of colonic distension pressure $[F(3,39)=415.6 ; P<0.0001]$, and a significant interaction between treatment and distension pressure $[F(3,39)=11.07 ; P<0.0001](n=7$ or 8 ; two-factor RM-ANOVA). For the maternal separation model, there was a significant effect of drug treatment $[F(1,10)=17.56 ; P=0.002]$, a significant effect of colonic distension pressure $[F(3,30)=$ 173.4; $P<0.0001$ ], and a significant interaction between treatment and distension pressure $[F(3,30)=7.61 ; P=0.001]$ ( $n=6$ per group; two-factor RM-ANOVA). Confirmation that the animal model behaved as expected was demonstrated with rats dosed with the known efficacious compound doxantrazole, a mast cell stabilizer (Fig. 5C) (van den Wijngaard et al., 2009). In the limited nesting model following doxantrazole treatment, there was a significant effect of drug treatment $[F(1,14)=26.96$; $P=0.0001]$, a significant effect of colonic distension pressure $[F(3,42)=269.2 ; P<0.0001]$, and a significant interaction between treatment and distension pressure $[F(3,42)=9.71$; $P<0.0001$ ] ( $n=8$ per group; two-factor RM-ANOVA).

\section{Discussion}

Visceral pain along with motility issues is the main concern for IBS patients that drives them to seek medical care. Currently, available treatments mainly offer relief from motility issues and do not adequately address visceral pain. Thus, there is an urgent need for drugs that can ameliorate visceral pain. Here, we sought to explore a role for RET in the control of IBS-like visceral sensitivity using a novel, potent, and selective RET inhibitor, GSK3179106. We demonstrated that the RET gene is expressed in thoracic and lumbosacral DRGs and that RET is functionally involved in visceral hypersensitivity. These 


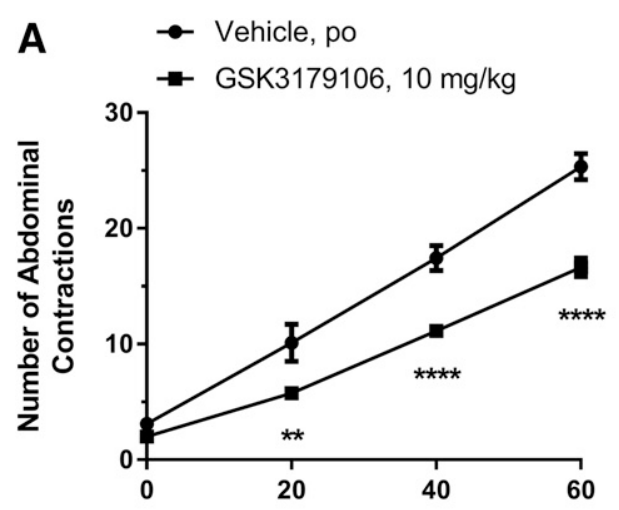

Colorectal Distension Pressure $(\mathrm{mmHg})$

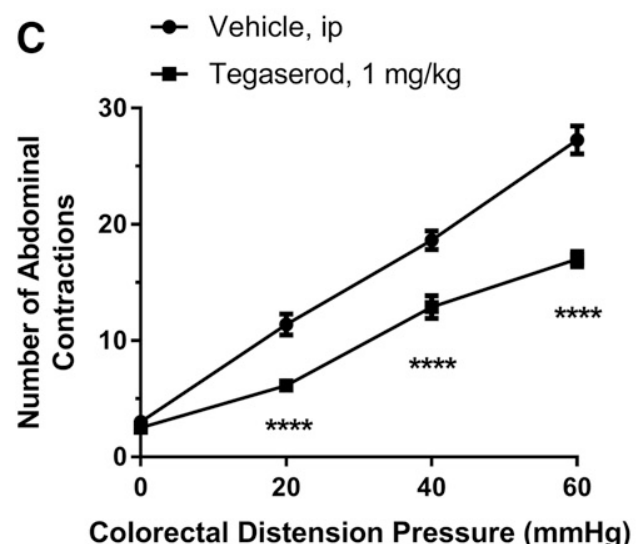

$\mathbf{E}$

-๘. Saline + Vehicle

-•· Saline + GSK3179106, 10 mg/kg

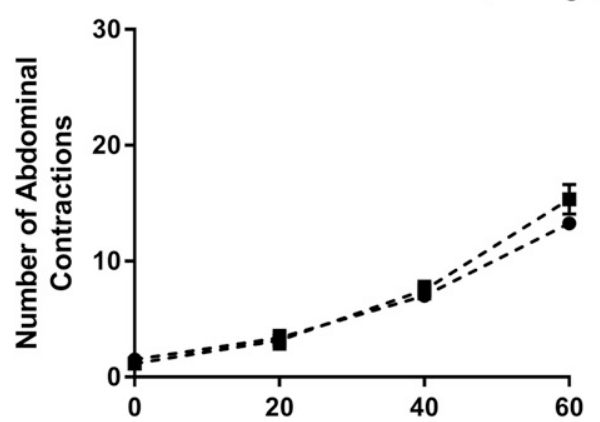

Colorectal Distension Pressure $(\mathrm{mmHg})$

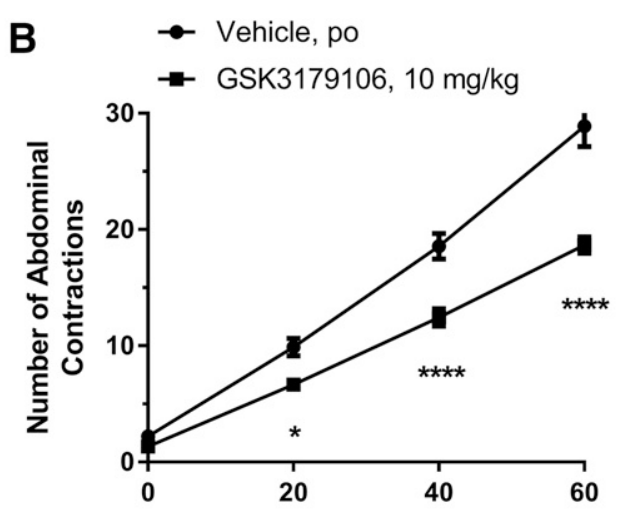

Colorectal Distension Pressure $(\mathrm{mmHg})$

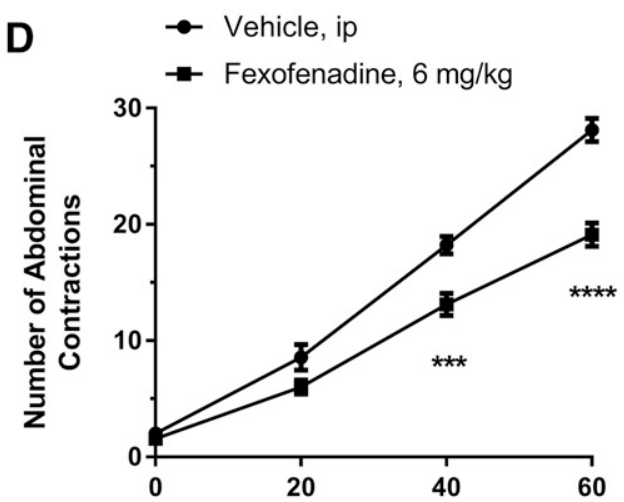

Colorectal Distension Pressure $(\mathrm{mmHg})$

F $\quad-\mathbf{m} \cdot$ Sham + Vehicle

-•· Sham + GSK3179106, 10 mg/kg

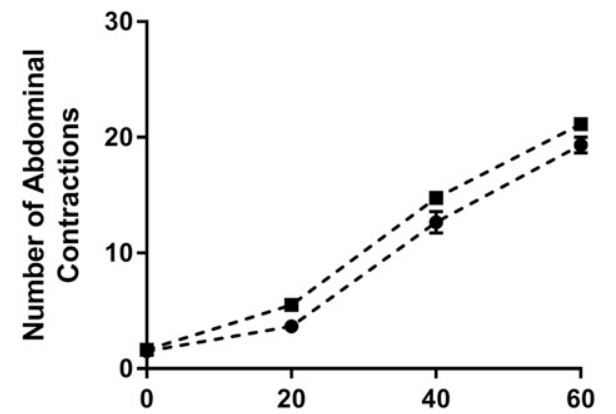

Colorectal Distension Pressure $(\mathrm{mmHg})$

Fig. 4. Colonic hypersensitivity induced by either postinflammatory stress or chronic adult stress is ameliorated by inhibition of RET kinase with GSK3179106. Rats dosed with GSK3179106 for 3.5 days twice a day exhibit an attenuated visceromotor response to colonic distension after colonic hypersensitivity was induced (A) upon the onset and resolution of acute colitis $(n=8)$ or (B) following exposure to WAS ( $n=9)$. (C) Rats with colonic hypersensitivity after the induction and resolution of acute colitis have a decreased visceral motor response upon intraperitoneal dosing with tegaserod $(n=8)$, a 5 - $\mathrm{HT}_{4}$ agonist, compared with vehicle-dosed rats $(n=8)$. (D) Rats with colonic hypersensitivity after exposure to WAS have a decreased visceral motor response upon oral dosing with fexofenadine, a histamine receptor 1 antagonist, compared with vehicle-dosed rats. (E) GSK3179106 dosed orally at $10 \mathrm{mg} / \mathrm{kg}$ for 3.5 days twice a day had no effect on the visceromotor response in sham-treated rats exposed to saline instead of TNBS $(n=8)$ in comparison with sham-treated rats dosed with vehicle $(n=6)$. (F) GSK3179106 dosed orally at $10 \mathrm{mg} / \mathrm{kg}$ for 3.5 days twice a day had no effect on the visceromotor response in sham-treated rats $(n=6)$ in comparison with sham-treated rats dosed with vehicle $(n=$ 8). ${ }^{*} P<0.05 ; * * P<0.01$; $* * * P<0.001$; $* * * P<0.0001$ vehicle compared with GSK3179106 treatment, two-way RM-ANOVA, and Bonferroni's post-test.

findings provide a link to the identity and function of RETexpressing neurons and to how RET inhibition may be of therapeutic value for the treatment of IBS-associated abdominal pain.
GSK3179106 is a potent, selective, and GI-restricted RET inhibitor that is minimally absorbed through the GI tract when dosed orally, resulting in low systemic bioavailability, and attenuates the visceromotor response in a preclinical 

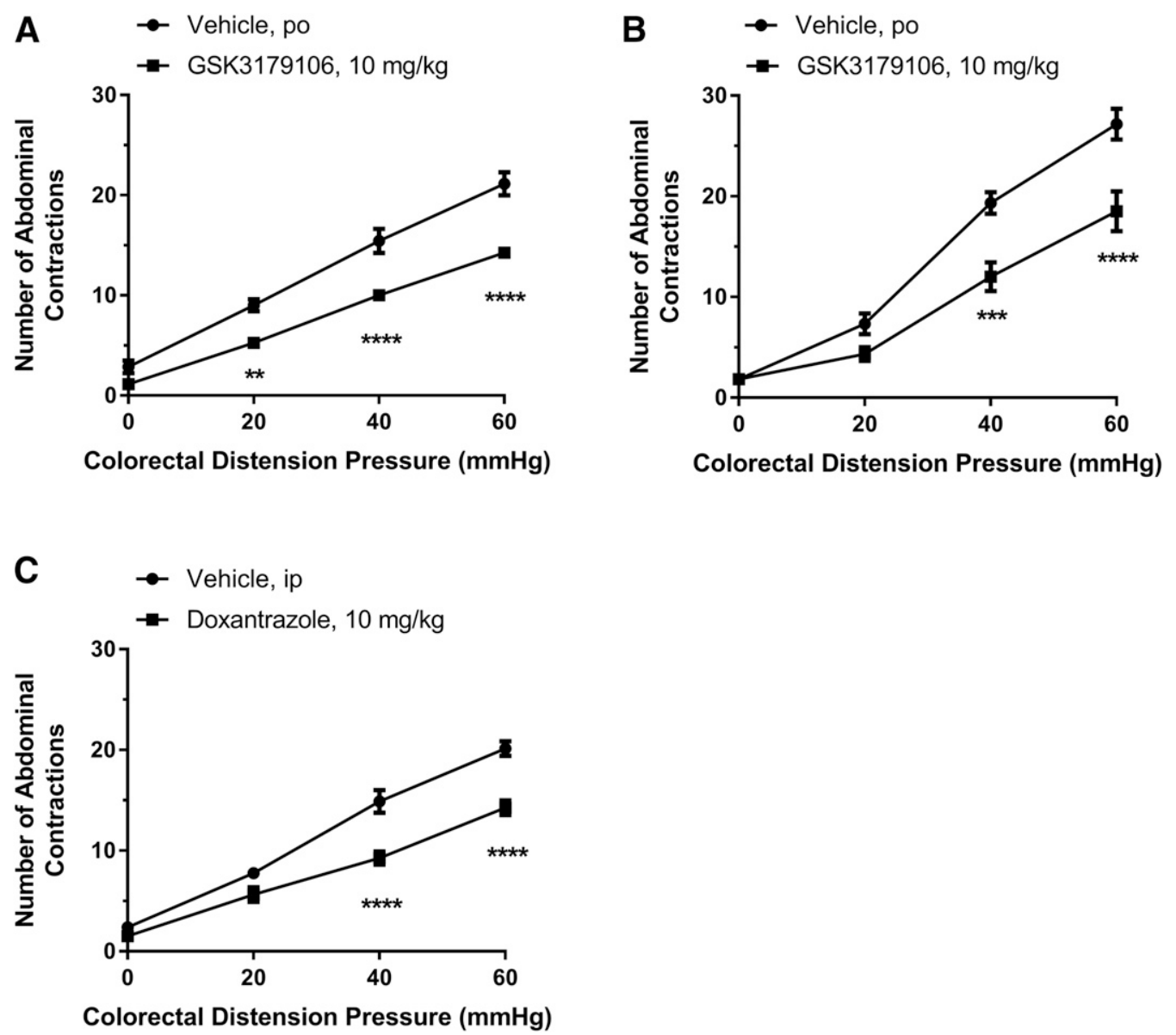

Fig. 5. Colonic hypersensitivity induced by either early life stress is ameliorated by inhibition of RET kinase with GSK3179106. Rats dosed with GSK3179106 for 3.5 days twice a day exhibit an attenuated visceromotor response to colonic distension after colonic hypersensitivity was induced by exposure of rats to limited nesting stress ( $n=7$ to 8/group), a form of early life stress (A), and by exposure to maternal separation ( $n=6$ /group), another form of early life stress (B). (C) In comparison with rats exposed to limited nesting stress and orally dosed with vehicle $(n=8)$, rats dosed with doxantrazole $(n=8)$, a mast cell stabilizer, have a reduced visceral motor response to colonic distension. ${ }^{*} P<0.01 ; * * * P<0.001 ; * * * * P<0.0001$ vehicle compared with GSK3179106 treatment, two-way RM-ANOVA, and Bonferroni's post-test.

model of acute visceral hypersensitivity (Schenck Eidam et al., 2018). We have expanded upon those findings and demonstrate that GSK3179106 is equally potent against the human and rat RET enzyme, supporting the conclusion that the efficacy observed in the in vivo colonic hypersensitivity models is due to RET inhibition. Furthermore, the compound demonstrates potent inhibition of RET phosphorylation in two cell lines that possess either a constitutively active or ligand-activated form of RET kinase. The reversible binding of the inhibitor to human RET kinase and the ability to potently inhibit the proliferation of RETdependent cell lines, while having no effect on the proliferation of RET-independent cell lines, demonstrates the selectivity of the inhibitor for RET and supports the idea that the findings in the in vivo model were not due to offtarget toxicity. These characteristics make GSK3179106 a useful tool for studying the effects of RET inhibition on colonic hypersensitivity in response to luminal distension and activation of extrinsic primary afferent nerves innervating the colon. Furthermore, GI restriction potentially provides for a higher therapeutic index and a better safety profile for an IBS drug by eliminating side effects known to occur with systemically available kinase inhibitors (Gharwan and Groninger, 2016).
In the current study of the DRG that project to the colon, RET-expressing neuronal cell bodies were found to coexpress CGRP, consistent with the suggestion that RET plays a role in extrinsic visceral afferent nociception. In light of this observation, we were interested in understanding if RET inhibition may influence the communication of visceral pain. Animal models of IBS recapitulate the symptoms of abdominal pain and altered bowel habits observed in human disease and therefore provide a useful tool in the understanding of the pathophysiology and the effects of therapeutic interventions (Holschneider et al., 2011; Greenwood-Van Meerveld et al., 2015; Moloney et al., 2015). Commonly, these studies are conducted in two phases; the first acts to sensitize afferent neurons, while the second, mediated by neurotransmission conducted via DRG, measures the visceromotor response (a surrogate for visceral pain perception) to colonic distension via analysis of abdominal contractions. We employed multiple previously validated experimental models to sensitize visceral afferent neurons to colonic distension. The rat models of colonic hypersensitivity included exposure to an acute colonic irritant, post-TNBS-induced colonic inflammation, exposure to chronic WAS, and neonatal early life stress induced by either limited nesting or maternal separation. In all of these models, the various manipulations resulted in an increased 
VMR to CRD compared with nontreated or sham-treated control animals. Inhibition of RET with GSK3179106 resulted in normalization of the VMR in response to CRD in hypersensitive animals regardless of the methodology used to induced colonic sensitization. Since the exposure that induced the hypersensitivity was different for each model, and in some cases applied prior to RET inhibition, it implies that RET inhibition affects the transmission and perception of the nociceptive signal associated with luminal distension rather than an indirect inhibition of the methodology used to produce the colorectal hypersensitivity. Furthermore, given the low systemic distribution of GSK3179106, we speculate that the compound directly affects visceral afferent neural processes innervating the colon rather than the cell bodies in DRG. Taken together, these data are compatible with the idea that RET-expressing DRG neurons that innervate the colon are part of a neuronal mechanosensation circuit that is activated upon colonic distension regardless of the neuronal sensitizing event.

In summary, the use of a potent, selective, and GI-restricted RET inhibitor, GSK3179106, has allowed the exploration of the role of RET function in the adult colon as a potential treatment of visceral pain. We identified RET-expressing neurons in thoracic and lumbosacral DRG and found that RET inhibition normalized the VMR to CRD in multiple rodent models of colonic hypersensitivity. Notably, RET antagonism normalized sensitized neurons regardless of the sensitizing method; however, RET antagonism had no effect on basal sensation. Although an understanding of the exact molecular mechanism by which RET inhibition affects visceral hypersensitivity in the models presented in the current study awaits further characterization, we speculate that RET activity may regulate the expression or function of proteins required to activate or maintain extrinsic primary afferent neuron signaling.

\section{Acknowledgments}

This work was supported by GlaxoSmithKline. We also thank Professor Hideki Enomoto M.D., Ph.D. (Graduate School of Medicine, Kobe University, Japan) for the use of the $\operatorname{Ret}^{\mathrm{CFP} /+}$ mice.

\section{Authorship Contributions}

Participated in research design: Russell, Mohammadi, Ligon, Gershon, Rao, DeMartino, Oliff, Kumar, Greenwood-Van Meerveld. Conducted experiments: Mohammadi, Ligon, Gershon, Rao, Shen, Chan.

Contributed new reagents or analytic tools: Eidam, Cheung.

Performed data analyses: Mohammadi, Johnson.

Wrote or contributed to the writing of the manuscript: Greenwood-Van Meerveld, Russell, Kumar, Gershon, Rao.

\section{References}

Airaksinen MS and Saarma M (2002) The GDNF family: signalling, biological functions and therapeutic value. Nat Rev Neurosci 3:383-394.

Azpiroz F, Bouin M, Camilleri M, Mayer EA, Poitras P, Serra J, and Spiller RC (2007) Mechanisms of hypersensitivity in IBS and functional disorders. Neurogastroenterol Motil 19 (Suppl 1):62-88.

Baloh RH, Enomoto H, Johnson EM Jr., and Milbrandt J (2000) The GDNF family ligands and receptors-implications for neural development. Curr Opin Neurobiol 10:103-110.

Borrello MG, Ardini E, Locati LD, Greco A, Licitra L, and Pierotti MA (2013) RET inhibition: implications in cancer therapy. Expert Opin Ther Targets 17: 403-419.

Brierley SM and Linden DR (2014) Neuroplasticity and dysfunction after gastrointestinal inflammation. Nat Rev Gastroenterol Hepatol 11:611-627.

Buhner S, Li Q, Berger T, Vignali S, Barbara G, De Giorgio R, Stanghellini V, and Schemann M (2012) Submucous rather than myenteric neurons are activated by mucosal biopsy supernatants from irritable bowel syndrome patients. Neurogastroenterol Motil 24:1134-e572.
Camilleri M (2012) Peripheral mechanisms in irritable bowel syndrome. $N$ Engl $J$ Med 367:1626-1635.

Dothel G, Barbaro MR, Boudin H, Vasina V, Cremon C, Gargano L, Bellacosa L, De Giorgio R, Le Berre-Scoul C, Aubert P, et al. (2015) Nerve fiber outgrowth is increased in the intestinal mucosa of patients with irritable bowel syndrome. Gastroenterology 148:1002-1011.e4.

Edery P, Lyonnet S, Mulligan LM, Pelet A, Dow E, Abel L, Holder S, Nihoul-Fékété C, Ponder BA, and Munnich A (1994) Mutations of the RET proto-oncogene in Hirschsprung's disease. Nature 367:378-380.

Gareau MG, Jury J, and Perdue MH (2007) Neonatal maternal separation of rat pups results in abnormal cholinergic regulation of epithelial permeability. Am J Physiol Gastrointest Liver Physiol 293:G198-G203.

Gharwan H and Groninger H (2016) Kinase inhibitors and monoclonal antibodies in oncology: clinical implications. Nat Rev Clin Oncol 13:209-227.

Greenwood-Van Meerveld B, Prusator DK, and Johnson AC (2015) Animal models of gastrointestinal and liver diseases. Animal models of visceral pain: pathophysiology, translational relevance, and challenges. Am J Physiol Gastrointest Liver Physiol 308:G885-G903.

Greenwood-Van Meerveld B, Venkova K, Hicks G, Dennis E, and Crowell MD (2006) Activation of peripheral 5-HT receptors attenuates colonic sensitivity to intraluminal distension. Neurogastroenterol Motil 18:76-86.

Halland M and Talley NJ (2013) New treatments for IBS. Nat Rev Gastroenterol Hepatol 10:13-23.

Holschneider DP, Bradesi S, and Mayer EA (2011) The role of experimental models in developing new treatments for irritable bowel syndrome. Expert Rev Gastroenterol Hepatol 5:43-57.

Ibáñez CF (2013) Structure and physiology of the RET receptor tyrosine kinase. Cold Spring Harb Perspect Biol 5:a009134.

Langlois A, Diop L, Rivière PJ, Pascaud X, and Junien JL (1994) Effect of fedotozine on the cardiovascular pain reflex induced by distension of the irritated colon in the anesthetized rat. Eur J Pharmacol 271:245-251.

Longstreth GF, Thompson WG, Chey WD, Houghton LA, Mearin F, and Spiller RC (2006) Functional bowel disorders. Gastroenterology 130:1480-1491.

Mayer EA (2008) Clinical practice. Irritable bowel syndrome. N Engl J Med 358: 1692-1699.

Moloney RD, O'Mahony SM, Dinan TG, and Cryan JF (2015) Stress-induced visceral pain: toward animal models of irritable-bowel syndrome and associated comorbidities. Front Psychiatry 6:15.

Plaza-Menacho I, Mologni L, and McDonald NQ (2014) Mechanisms of RET signaling in cancer: current and future implications for targeted therapy. Cell Signal 26:1743-1752. Plourde V, St-Pierre S, and Quirion R (1997) Calcitonin gene-related peptide in viscerosensitive response to colorectal distension in rats. Am J Physiol 273:G191-G196.

Polverino A, Coxon A, Starnes C, Diaz Z, DeMelfi T, Wang L, Bready J, Estrada J, Cattley R, Kaufman S, et al. (2006) AMG 706, an oral, multikinase inhibitor that selectively targets vascular endothelial growth factor, platelet-derived growth factor, and kit receptors, potently inhibits angiogenesis and induces regression in tumor xenografts. Cancer Res 66:8715-8721.

Prusator DK and Greenwood-Van Meerveld B (2015) Gender specific effects of neonatal limited nesting on viscerosomatic sensitivity and anxiety-like behavior in adult rats. Neurogastroenterol Motil 27:72-81.

Rao M, Nelms BD, Dong L, Salinas-Rios V, Rutlin M, Gershon MD, and Corfas G (2015) Enteric glia express proteolipid protein 1 and are a transcriptionally unique population of glia in the mammalian nervous system. Glia 63: 2040-2057.

Sariola H and Saarma M (2003) Novel functions and signalling pathways for GDNF. J Cell Sci 116:3855-3862.

Schenck Eidam H, Russell J, Raha K, DeMartino M, Qin D, Guan HA, Zhang Z, Zhen G, Yu H, Wu C, et al. (2018) Discovery of a first-in-class gut-restricted RET kinase inhibitor as a clinical candidate for the treatment of IBS. ACS Med Chem Lett $\mathbf{9}$ : 623-628.

Schwetz I, McRoberts JA, Coutinho SV, Bradesi S, Gale G, Fanselow M, Million M, Ohning G, Taché Y, Plotsky PM, et al. (2005) Corticotropin-releasing factor receptor 1 mediates acute and delayed stress-induced visceral hyperalgesia in maternally separated Long-Evans rats. Am J Physiol Gastrointest Liver Physiol 289: G704-G712.

Spencer NJ, Kyloh M, and Duffield M (2014) Identification of different types of spinal afferent nerve endings that encode noxious and innocuous stimuli in the large intestine using a novel anterograde tracing technique. PLoS One 9:e112466.

Spiller R and Garsed K (2009) Postinfectious irritable bowel syndrome. Gastroenterology 136:1979-1988.

Stanisor OI, van Diest SA, Yu Z, Welting O, Bekkali N, Shi J, de Jonge WJ, Boeckxstaens GE, and van den Wijngaard RM (2013) Stress-induced visceral hypersensitivity in maternally separated rats can be reversed by peripherally restricted histamine-1-receptor antagonists. PLoS One 8:e66884.

Tanaka T, Shinoda M, Feng B, Albers KM, and Gebhart GF (2011) Modulation of visceral hypersensitivity by glial cell line-derived neurotrophic factor family receptor $\alpha-3$ in colorectal afferents. Am J Physiol Gastrointest Liver Physiol 300: G418-G424.

Uesaka T, Nagashimada M, Yonemura S, and Enomoto H (2008) Diminished Ret expression compromises neuronal survival in the colon and causes intestinal aganglionosis in mice. J Clin Invest 118:1890-1898.

van den Wijngaard RM, Klooker TK, Welting O, Stanisor OI, Wouters MM, van der Coelen D, Bulmer DC, Peeters PJ, Aerssens J, de Hoogt R, et al. (2009) Essential role for TRPV1 in stress-induced (mast cell-dependent) colonic hypersensitivity in maternally separated rats. Neurogastroenterol Motil 21:1107-e94.

Address correspondence to: Dr. Beverley Greenwood-Van Meerveld, O'Donoghue Building, Rm 332, 1122 NE 13th St., Oklahoma City, OK 73117. E-mail: beverley-greenwood@ouhsc.edu 